EchoGéo

ECHOGEO-Sur le Vif | 2009

\title{
La diaspora tamoule en France : entre visibilité et politisation
}

\section{Anthony Goreau-Ponceaud}

\section{OpenEdition}

Journals

Édition électronique

URL : http://journals.openedition.org/echogeo/11157

DOI : 10.4000/echogeo. 11157

ISSN : 1963-1197

\section{Éditeur}

Pôle de recherche pour l'organisation et la diffusion de l'information géographique (CNRS UMR 8586)

Référence électronique

Anthony Goreau-Ponceaud, «La diaspora tamoule en France : entre visibilité et politisation », EchoGéo [En ligne], Sur le Vif, mis en ligne le 13 mai 2009, consulté le 10 décembre 2020. URL : http:// journals.openedition.org/echogeo/11157; DOI : https://doi.org/10.4000/echogeo.11157

Ce document a été généré automatiquement le 10 décembre 2020.

\section{(c) (i) (9)}

EchoGéo est mis à disposition selon les termes de la licence Creative Commons Attribution - Pas d'Utilisation Commerciale - Pas de Modification 4.0 International 


\title{
La diaspora tamoule en France : entre visibilité et politisation
}

\author{
Anthony Goreau-Ponceaud
}

\section{Introduction}

1 Cette analyse pourrait commencer par l'évocation d'un proverbe tamoul qui à lui seul résume les enjeux de la constitution d'un espace diasporique : «tout est mon pays, tout est ma parenté » (« Kanniyan Poongundran »). En effet, on estime la diaspora tamoule à plus de 8.4 millions d'individus dispersés dans le monde, soit un peu moins de $40 \%$ de ce que l'on appelle communément la diaspora indienne (Goreau-Ponceaud, 2008). La population tamoule étant établie un peu partout dans le monde, il devient impossible pour ses membres d'identifier leur territoire («tout est mon pays»), car celui-ci s'étend peu à peu au globe dans son intégralité. La mobilité ne peut plus être qu'une mobilité du dedans, à l'intérieur d'un corps social devenu territoire (" tout est ma parenté »). De sorte que l'espace de la diaspora est partout et nulle part à la fois. Pourtant, derrière cette illusion communautaire renforcée par le pouvoir des chiffres, apparaissent de multiples divergences. La diaspora tamoule n'est pas un ensemble monolithique. Elle est divisée non seulement par des clivages prémigratoires liés à la caste, à la classe sociale, à la religion, au genre, au village et à la région d'origine ; mais aussi par des distinctions provenant des différents processus ou contextes migratoires qui ont de larges implications dans la détermination du statut des Tamouls dans le pays d'accueil : tantôt clandestins, réfugiés, immigrés ou encore citoyens.

2 Ainsi au-delà de ce souci d'universalité affiché, comment définir l'identité tamoule ? Comment passe-t-on de l'idée d'une langue commune - le tamoul - à un peuple en diaspora? Ce savoir-être d'ici et de là-bas produit-il d'originales constructions identitaires et territoriales? En somme, ce proverbe soulève fondamentalement la question de l'habiter et de l'identité. Il pose comme problématique l'existence d'une communauté à l'étranger, prise en étau par une triple ambivalence: entre ancrage et 
mobilité, entre ouverture et fermeture et entre identité racine - exclusive de l'autre - et identité rhizome qui se déroule vers la rencontre avec l'autre (Glissant, 1996).

Après avoir défini brièvement ce que l'on entend par monde tamoul, nous focaliserons notre attention sur un groupe particulier: les Tamouls originaires de Sri Lanka. Forte d'une communauté de plus de 70000 personnes, leur arrivée en France à partir des années 1970 a généré de multiples transformations souvent perçues par les autres Tamouls (de Pondichéry, du monde caribéen ou indianocéanique) comme l'intrusion d'un possible désordre au cœur d'un paysage social et culturel qu'ils regardaient jusque là comme homogène ou plutôt serein. Ces derniers se sont vus progressivement assimilés à ces « nouveaux entrants » arrivés de manière illicite, bousculant ainsi leur appartenance à la catégorie des «vieux nationaux $»^{1}$. Cet article se propose d'analyser ces transformations en portant une attention toute particulière à l'une des originalités de ce dernier segment migratoire: sa politisation et sa demande de reconnaissance qui passe par la visibilité.

\section{Du monde tamoul à l'identité tamoule}

4 Au préalable, il est nécessaire de s'interroger sur la légitimité qu'il y a à parler de diaspora dans le cas des Tamouls. Pour répondre à cette première interrogation il faut revenir sur l'élément qui est à la genèse de cette réflexion: le festival de Ganesh, dont l'origine est à rechercher du côté d'un miracle.

5 Le 21 septembre 1995 en Inde comme dans tous les pays où résident des Indiens, les dieux se sont manifestés ouvertement et ont bu le lait qui leur avait été offert en oblation. « Le miracle pu être constaté partout où se trouvaient les statues de Ganesh et des divinités associées au dieu Shiva : que ce soit dans les temples ou chez les particuliers et aussi bien en Inde que dans le monde entier; partout en fait où il y avait des dévots ou même des simples curieux qui voulaient en faire l'expérience » (Vidal, $1997: 884$ ). Depuis 1996 en l'honneur du dieu à tête d'éléphant, un temple hindou du $18^{\mathrm{e}}$ arrondissement de Paris - le temple de Ganesh ou association Sri Manika Vinayakar Alayam - organise une fête tous les ans.

6 Cet évènement soulève plusieurs interrogations qui constituent la base de notre questionnement : qu'est-ce qui se donne à voir, là, sous nos yeux dans les rues de Paris? Comment penser ce collectif? Et que vise la communauté tamoule par cette visibilité ? 
Photo 1 - Le festival de Ganesh

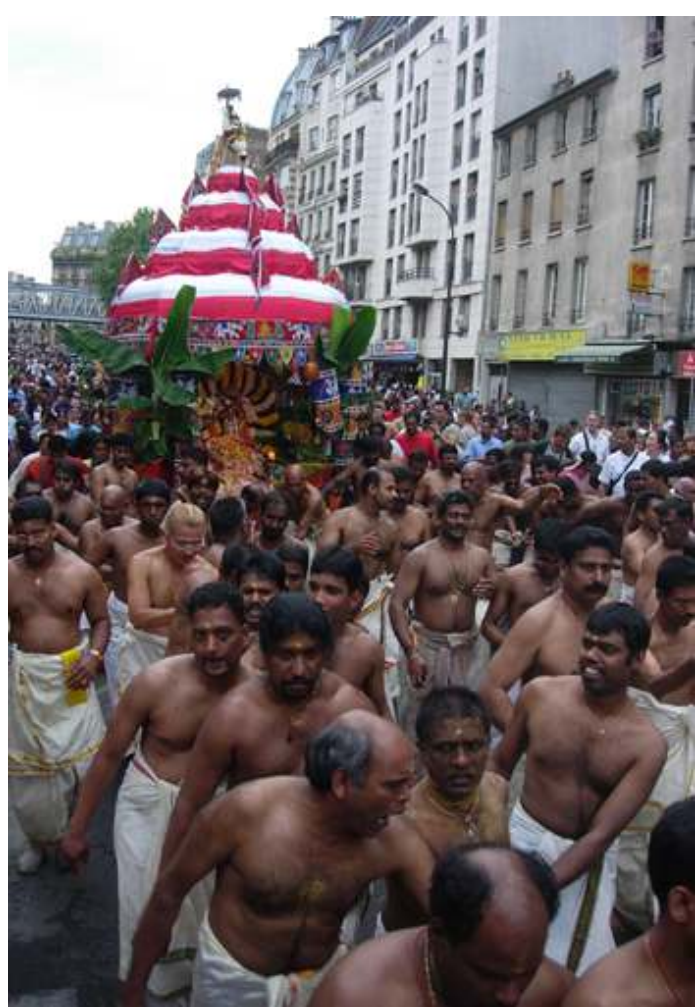

Ce festival se déroule en France le dernier dimanche d'août ou le premier du mois de septembre (selon la date fixée par les astrologues indiens). À cette occasion, les hindous d'île-de-France organisent un défilé où le char du dieu à tête d'éléphant est promené dans les rues voisines du temple, situé dans le $18^{\mathrm{ème}}$ arrondissement,au 72 rue Philippe de Girard. Au cours du trajet, le cortège, composé de danseurs et de danseuses portant sur leurs épaules des arceaux de plumes de paon et sur leur tête des pots de terre cuite, passe devant des tas de noix de coco qui sont alors brisées et distribuées. Vient ensuite le char de Ganesh mesurant près de $5 \mathrm{~m}$ de haut et tiré par des hommes avec l'aide de 2 cordes de $20 \mathrm{~m}$ de long.

7 En observant et en discutant avec quelques acteurs de cette fête, nous nous sommes rendus compte que les prêtres qui officiaient venaient pour certains d'entre eux de l'île de la Réunion, d'autres de Rameshwaram (ville sainte du Tamil Nadu), que la statue de Ganesh provenait du Sri Lanka et qu'elle avait été bénie par un Anglais! Mais plus surprenant encore, lorsque l'on demandait aux personnes assistant à cette fête qui en était l'organisateur, on ne nous répondait pas le temple de Ganesh, mais la communauté tamoule. Plus précisément la communauté tamoule du Sri Lanka. Quant aux spectateurs, à la question « d'où venez-vous? », au lieu de nous répondre de Sarcelles, La Courneuve ou Paris (leur lieu de résidence), ils disaient de Pondichéry, de Maurice, de la Réunion ou du Sri Lanka (leur lieu d'origine). Et quand on les interrogeait à propos du motif de leur présence, ils nous répondaient soit "parce que nous sommes tamouls», soit "parce que nous sommes hindous » et souvent « pour maintenir notre identité »².

8 Ainsi, si l'on s'accorde à une définition minimaliste de la diaspora (Cohen,1997), en tant que l'ensemble des membres d'un collectif dispersés à travers le monde mais restant en relation les uns avec les autres, cet évènement met en lumière la notion de diaspora. D'autant plus que l'on a bien à faire à un collectif dispersé. Certes ce collectif ne constitue pas un ensemble homogène. Mais il y a tout de même dispersion à partir de deux pôles, à partir de deux référents-origines : le Tamil Nadu et le Nord Sri Lanka, qui tous deux sont 
profondément marqués par la culture tamoule. Et, même si les Tamouls sri lankais sont investis d'un sentiment insulaire qui s'est souvent traduit par un déni d'indianité (Meyer, 2001), la liste des points communs que l'on peut faire entre ces différents groupes est importante : la langue, le système de valeur et la religion ${ }^{3}$.

\section{Il y a tamoul et tamouls}

Nous avons donc individualisé les Tamouls d'Inde des Tamouls du Nord Sri Lanka, au référent-origine distinct : le Tamil Nadu, et le Tamil Eelam4.

Figure 1 - La diaspora tamoule

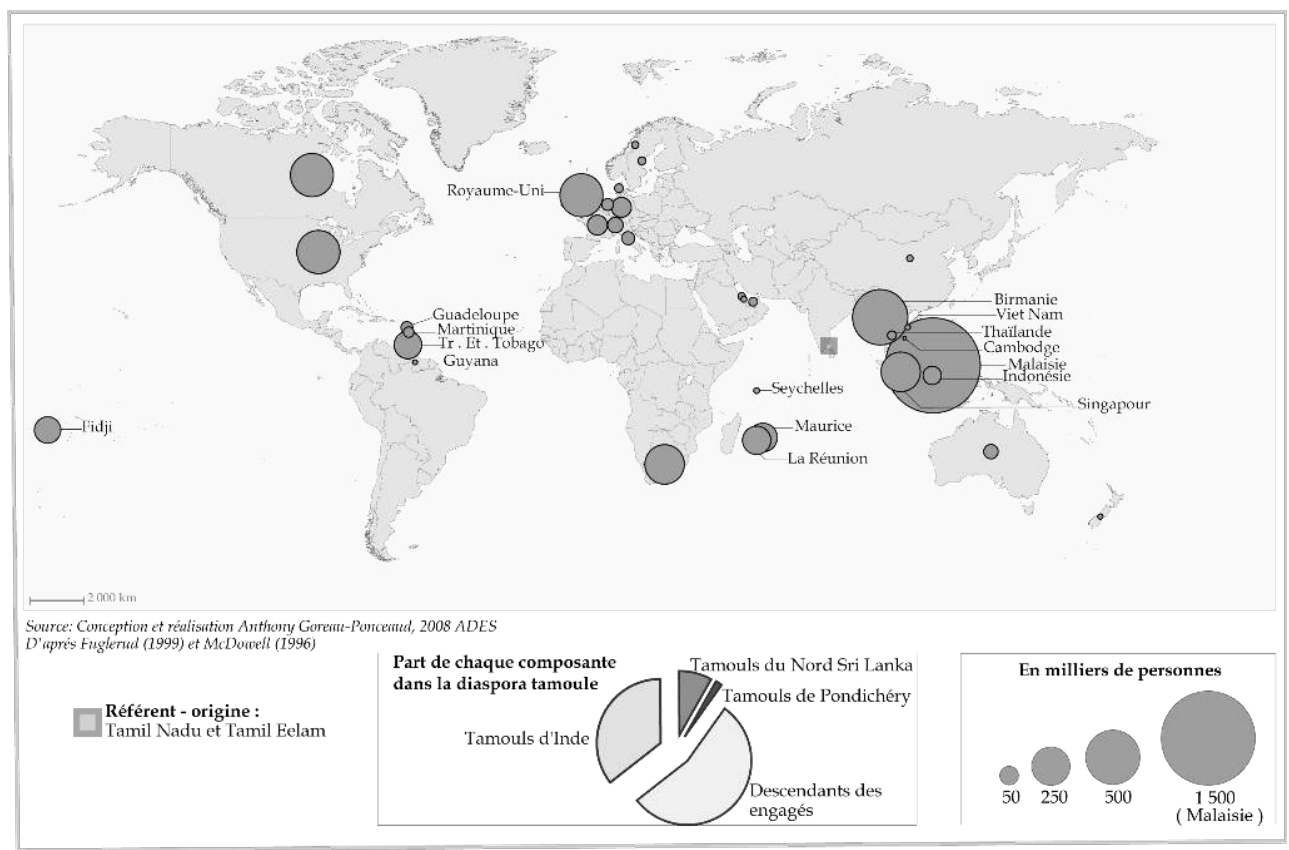

Les contextes de mise en place des cadres migratoires et les trajets sont aussi différents. D'un côté, les Tamouls d'Inde se sont dispersés massivement durant la période coloniale (de 1834 à 1914) dans le cadre d'une émigration de travailleurs sous contrat, communément appelée système de l'indenture. De l'autre, les mouvements migratoires contemporains sont plutôt constitués de réfugiés tamouls provenant du Nord Sri Lanka. Le contexte n'est plus celui de la période coloniale : c'est dans l'émergence d'une guerre civile, dans les années 1970, opposant la majorité cinghalaise, bouddhiste ( $74 \%$ de la population), à la minorité tamoule (12.6 \% de la population), hindoue, que cette migration s'est intensifiée (Meyer, 2001). Néanmoins, il est indispensable de préciser que si les Tamouls Sri lankais ont fait le choix de s'installer en France à partir de la fin des années 1970, début des années 1980, c'est que la plupart ne pouvaient plus se rendre en Angleterre du fait du durcissement des lois sur l'immigration, et stoppaient leur voyage en France, arrivant à Paris par la Gare du Nord. C'est comme cela que l'on a assisté, entre les stations de métro la Chapelle et Gare du Nord, à la constitution progressive d'un entre soi. Et si l'on tient compte de la morphologie du quartier, cet entre-soi que les Tamouls appellent « la Chapelle », a toutes les caractéristiques d'une porte d'entrée. Inséré entre deux gares (Gare de l'Est et Gare du Nord), limitrophe au Nord des quartiers immigrés d'implantations plus anciennes que sont Barbès, Château Rouge et la Goutte d'Or, offrant 
une importante concentration d'hôtels modestes et peu coûteux, ce quartier semble proposer, plus qu'ailleurs, des facilités pour s'établir seul, sans relais communautaire.

De ce fait, il semble important de distinguer au sein de cette migration deux grandes phases qui tiennent compte de la constitution progressive des chaînes migratoires. Aux premiers migrants, des années 1970-1980, constituant le premier noyau de demandeurs d'asiles sri lankais en France, s'opposent ceux des années 1990-2000. En effet, entre 1979 et 1983, les Sri Lankais continuent à arriver en France mais s'y trouvent dans une situation très précaire. Le conflit au pays n'a pas encore été vraiment reconnu par l'administration française et presque tous sont déboutés dans leur demande d'asile.

Tableau 1 - Les demandeurs d'asile sri lankais en Europe occidentale entre 1990 et 1999

\begin{tabular}{|l|c|c|c|}
\hline Année & $\begin{array}{c}\text { Nombre de } \\
\text { demandes d'asile } \\
\text { provenant du Sri } \\
\text { Lanka }\end{array}$ & $\begin{array}{c}\text { Total des } \\
\text { demandeurs d'asile } \\
\text { (toutes nationalités } \\
\text { confondues) }\end{array}$ & $\begin{array}{c}\text { \% de la demande } \\
\text { d'asile sri-lankaise sur } \\
\text { I'ensemble. }\end{array}$ \\
\hline 1990 & 19300 & 424400 & 4.55 \\
1991 & 23700 & 526300 & 4.5 \\
1992 & 16800 & 684800 & 2.45 \\
1993 & 12600 & 545700 & 2.31 \\
1994 & 12900 & 318500 & 4.05 \\
1995 & 12700 & 319900 & 3.98 \\
1996 & 12400 & 276200 & 4.49 \\
1997 & 13000 & 312900 & 4.15 \\
1998 & 11100 & 334300 & 3.32 \\
1999 & 11400 & 412700 & 2.76 \\
Total & 145900 & 4155700 & 3.51 \\
\hline
\end{tabular}

Source : Les réfugiés dans le monde. 50 ans d'action humanitaire. Haut commissariat des Nations Unies pour les réfugiés. Annexe 10. 2000

12 À la suite des violents évènements de Colombo en 1983 (Black July Pogrom) et jusqu'en 1987, l'afflux de Tamouls redouble. Des femmes avec leurs enfants commencent à rejoindre des maris qui n'ont pas encore de réponse à leur demande d'asile. C'est alors la période où dans toute l'Europe s'installe le débat sur les réfugiés politiques/réfugiés économiques, et qu'en France, les associations, les organismes d'accueil, ou la presse s'interrogent sur la gravité réelle de la situation des Tamouls à Sri Lanka autant que sur la précarité de leur condition de vie sur le territoire français (Goreau-Ponceaud, 2008). Ainsi, en l'espace d'une décennie en Europe, plus de 145000 Sri Lankais ont demandé l'asile. Les demandes atteignent leur paroxysme en 1991. À partir de 1988, la reconnaissance du statut de réfugié aux Tamouls devient quasiment systématique. Fin 1991, l'OFPRA (Office Français de Protection des Réfugiés et des Apatrides) régularise même sous ce statut tous les dossiers en attente. Quant aux déboutés (rejetés du droit d'asile), ils ont le plus souvent bénéficié de plusieurs autres mesures de régularisation avec un statut de travailleur immigré. Les Sri Lankais constituent donc jusqu'en 1995, une des premières nationalités à se faire reconnaitre le statut de réfugié 5 . 
Tableau 2 - Demandes d'asile des sri lankais en France et pourcentage d'accord

\begin{tabular}{|c|c|c|c|}
\hline Année & $\begin{array}{c}\text { Première demande d'asile } \\
\text { des sri lankais hors mineurs } \\
\text { accompagnants. }\end{array}$ & $\begin{array}{c}\text { Part de premières demandes } \\
\text { des sri lankais par rapport à } \\
\text { Pensemble des premières } \\
\text { demandes. }\end{array}$ & \% d'accord \\
\hline 1996 & 1169 & $8.2 \%$ & \\
1997 & 1582 & $9.0 \%$ & \\
1998 & 1832 & $8.2 \%$ & $43.2 \%$ \\
1999 & 2001 & $6.5 \%$ & $43.2 \%$ \\
2000 & 1879 & $4.8 \%$ & $33.5 \%$ \\
2001 & 2000 & $4.2 \%$ & $22,0 \%$ \\
2002 & 1992 & $3.9 \%$ & $14.0 \%$ \\
2003 & 1967 & $3.8 \%$ & $9.9 \%$ \\
2004 & 2090 & $4.1 \%$ & $4.9 \%$ \\
2005 & 1894 & $4.4 \%$ & $5.1 \%$ \\
2006 & 1993 & $7.6 \%$ & \\
\hline
\end{tabular}

Source : D'après les rapports d'activité de l'OFPRA. Aujourd'hui le quartier ne compte qu'un faible pourcentage de résidents tamouls. La constitution des chaînes migratoires a donné lieu à une dilution résidentielle des Tamouls dans les communes Nord (Sarcelles, Garges-lès-Gonesse, La Courneuve, Villiers-le-Bel, le Bourget, le Blanc-Mesnil, Saint-Denis, Aubervilliers) et Est (Montreuil, Chelles, BoissySaint-Léger) de la périphérie parisienne. Des communes qui sont toutes connectées à la Chapelle via le réseau des transports franciliens (RER et métro).

Entre ces deux phases les routes empruntées divergent elles aussi: elles se sont multipliées au grès des bouleversements géopolitiques. À la simplicité des routes terrestres des années 1970-1980 (où l'entrée sur le continent européen se faisait via l'Ukraine et la Turquie), s'oppose la complexité des routes d'après 1991. Les pays traversés deviennent plus nombreux, amplifiant la durée du trajet et le nombre de passages (Goreau-Ponceaud, 2008).

Texte 1 - Un trajet qui se complexifie (extrait d'un entretien mené à Paris en 2006)

-Interlocuteur : «J'ai quitté le Sri Lanka en 1996. Pour venir en France cela m'a pris plusieurs mois. Approximativement seize mois. D'abord je suis parti de Talaimannar au Sri Lanka pour rejoindre l'Inde. J'ai pris un bateau de nuit. Je suis arrivé prés de 
Danuskkodi ; ce n'est pas très loin de Rameswaram7 , c'est de l'autre côté du détroit qui sépare mon île de l'Inde. C'est la route la plus courte pour aller d'un côté à l'autre. Il n'y a qu'à embarquer dans un des nombreux bateaux qui font la navette la nuit. De là je suis allé à Pondichéry. Là-bas, un ami que je connaissais m'a arrangé le coup. J'ai pu prendre l'avion pour l'Afrique du Nord. Après j'ai pris le bateau, direction l'Espagne. De là, j'ai repris un avion avec lequel je suis arrivé aux Pays-Bas et j'ai finalement pris un bus pour la France ».

-Interviewer : « Et pourquoi la France? ».

-Interlocuteur: «Parce que j'y connaissais du monde qui pouvait m'accueillir et m'obtenir un emploi. C'est pour cette raison que j'ai voulu aller en France ».

Parallèlement à ces deux segments, la présence française à Pondichéry (de 1671 à 1962) a contribué à un brassage des cultures tamoule et française, et à la formation d'un cycle migratoire vers la France qui se poursuit aujourd'hui encore. Une partie de ces Français d'origine pondichérienne, les Marécars ${ }^{8}$, qui avaient migré après la seconde guerre mondiale vers l'Indochine, puis repartis en France, aux Mascareignes, en Afrique subsaharienne, et en Asie du Sud-Est après la chute de Saigon en 1975, a servi de pivot aux trajectoires tamoules sri lankaise et à leur installation en Île-de-France dans les années 1970.

Malgré tout, cette dispersion n'est pas suffisante pour désigner les Tamouls en tant que diaspora, puisque selon la définition vue ci-dessus, il faut que les membres de ce collectif restent en relation les uns avec les autres. En somme, comment la diversité d'origine spatiale et temporelle des Tamouls en France peut-elle se fondre en une identité reconstituée par la diaspora? Plus précisément, quels points de condensation localisés ou thématisés (le religieux, le politique et la langue) produisent une unité alors que trois groupes d'origine peuvent être reconstitués:les Franco-Indiens de Pondichéry, les Tamouls ou Indiens descendants des engagés dispersés dans l'Empire colonial français et les Tamouls sri lankais réfugiés en France?

Du Nord Sri Lanka à la Courneuve : lieux et territoires tamouls en Île-de-France.

Dans Les cadres sociaux de la mémoire, Halbwachs (1994) établit que la pensée sociale est essentiellement une mémoire et que, par ailleurs, pour durer, celle-ci doit s'attacher à quelques points du sol. En d'autres termes, c'est par les lieux que la convergence de l'identité se produit. Mais, comment faire pour être ici et là-bas, l'un et l'autre à la fois ? Cela est affaire de proximité. Bien sûr il ne s'agit pas d'être à côté de, mais connecté à. Connecté à l'ailleurs et à là-bas via les moyens de communications, mais surtout via des liens. C'est ainsi que l'on assiste, dans ce nœud que constitue la métropole parisienne, à la construction et à la consolidation de territoires ethniques dont les symboles et l'iconographie sont les marqueurs. 
Figure 2 - Structures d'une diaspora

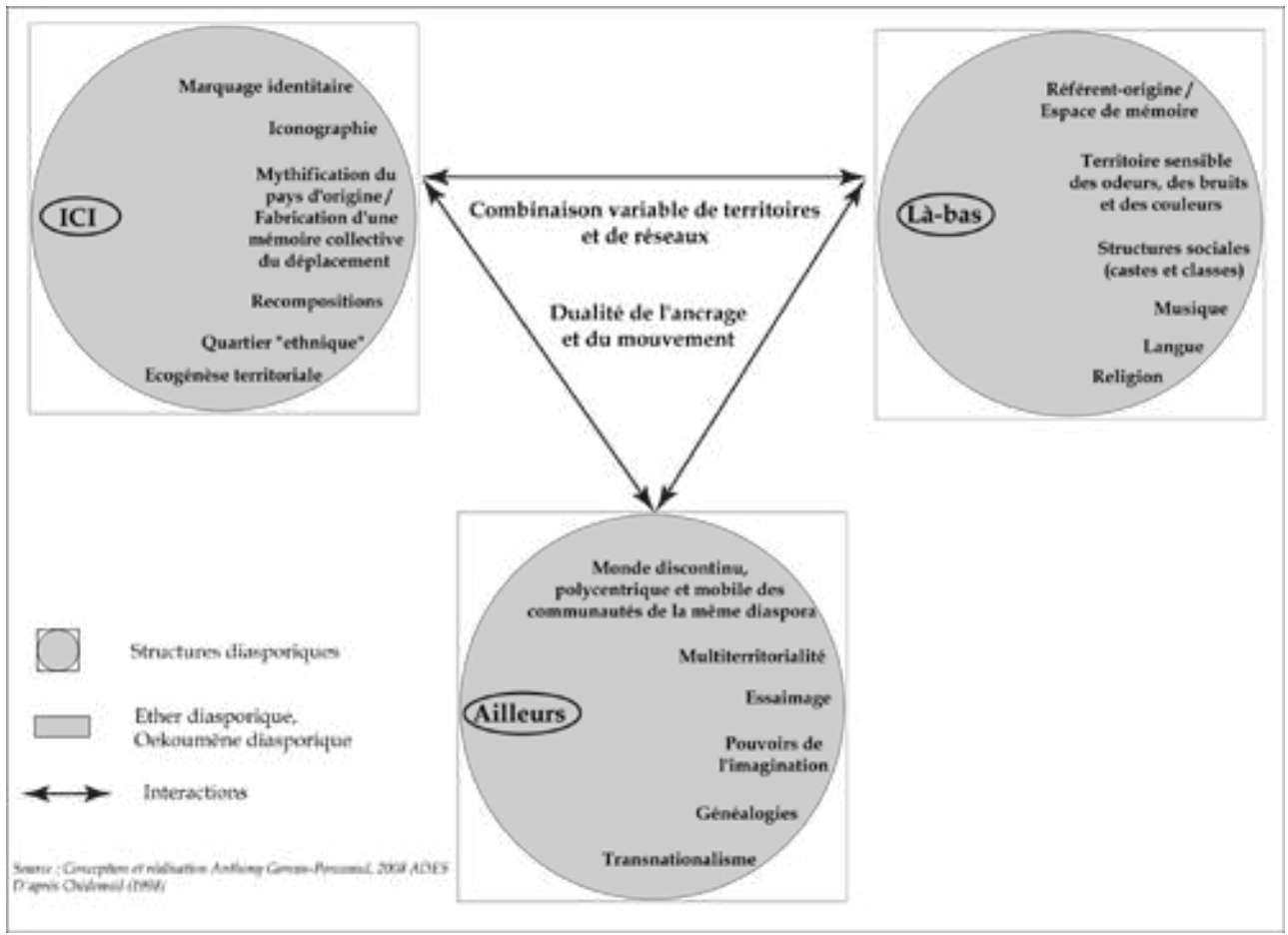

21 L'existence de la diaspora tamoule suppose des espaces d'ancrage indispensables à la survie de la communauté dans le territoire d'installation, qui sont rendus visibles par la présence d'objets concrets, ratifiés individuellement et/ou collectivement. La fracture entre là-bas et ailleurs, est compensée par la constitution en France de territoires qui évoquent l'existence de principes structurants et identificatoires, des marqueurs territoriaux et des « lieux de mémoires » (Nora, 1997) assurant le lien avec là bas. 


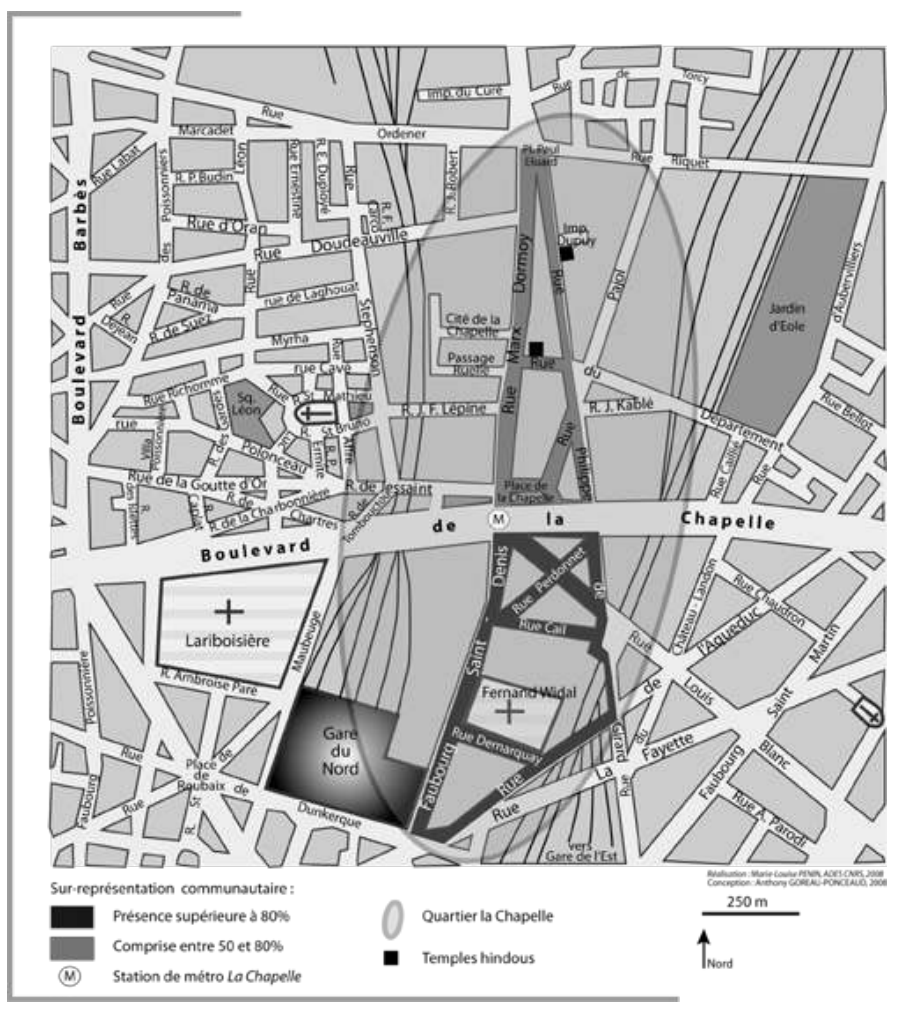

Ainsi, l'arrivée massive des Tamouls sri lankais en France à partir des années 1990 a mis en évidence la création d'ethnoterritoires (Dufoix\&Foucher, 2007). La Chapelle - ce quartier compris entre les $10^{\mathrm{e}}$ et $18^{\mathrm{e}}$ arrondissements de Paris - n'est pas seulement un port de première entrée défini en tant que tel par la solidité des chaînes migratoires, mais également le cœur d'un vaste réseau de solidarité communautaire. Cette centralité minoritaire (Raulin, 2000) remplit des fonctions économiques, commerciales, identitaires et politiques pour la communauté tamoule et plus particulièrement pour la communauté tamoule du Sri Lanka. On y recense deux temples hindous (complétés de onze autres dans les communes d'île-de-France), plusieurs écoles de tamoul (dites aussi tamoulcholaï) et de bharata natyam (danse classique originaire du sud de l'Inde) et 178 commerces (GoreauPonceaud, 2008). On a affaire ici à un « commerce ethnique » qui vise comme clientèle la communauté tamoule dont est issu le commerçant. Le soin apporté à l'intérieur comme à l'extérieur des différents magasins ainsi que les produits vendus manifestent explicitement cette appartenance communautaire. En effet, la majorité des enseignes reprennent des noms et des éléments culturels familiers aux Tamouls. De plus, ce commerce est souvent considéré par la communauté, comme un moyen de contourner les obstacles d'un marché du travail discriminatoire, et joue un rôle important dans les stratégies de positionnement identitaire.

Tout cela concourt à une mise en scène de l'altérité qui parait jouer une double fonction : elle structure l'identité pour soi, en articulant entre eux des aspects tout à fait intimes de la vie communautaire comme elle offre des points de repères stables - voire stéréotypés dans la présentation de soi pour les autres.

La visibilité de ce little India a eu d'importantes conséquences au niveau de la représentation de la catégorie « tamoule » qu'ont les membres de la société française. On 
doit prendre en considération que tous les groupes n'ont pas la même autorité pour se nommer et se faire nommer. N'oublions pas que la visibilité désigne bien plus que la perceptibilité parce qu'elle implique une capacité d'identification individuelle élémentaire (Honneth, 2006, 228). C'est ainsi qu'est apparue à partir des années 2000 une dissonance entre Tamouls indiens et Tamouls Sri Lankais au niveau du régime de visibilité. La relation intersubjective entre ces deux groupes s'est réglée par une incompatibilité entre les déterminations modales des deux parties. En effet, les Sri Lankais, du point de vue des Tamouls indiens, et en particulier Pondichériens, s'exposent davantage au regard de la société française. Cette ostentation, même si elle permet, via ces installations communautaires, une identité renaissante, les a fait sortir de l'anonymat. Du fait combiné de cette ostentation et de leur proximité phénotypique, les Français d'origine pondichérienne, qui se pensaient bien intégrés - bons Français et bons chrétiens - se sont vus progressivement assimilés aux Tamouls sri lankais (GoreauPonceaud, 2008).

\section{Diaspora et mobilisation politique}

Les Tamouls sri lankais par leur important tissu associatif et commercial, apparaissent comme des entrepreneurs d'identité locale (aux branchements transnationaux), mais la définition qu'ils en donnent ne fait pas consensus. La fonction de cette centralité minoritaire n'est pas la même selon que l'on se place du côté des Tamouls du Sri Lanka ou de celui des Tamouls d'Inde. Cette distinction tient compte de la particularité et de la contemporanéité des conditions de l'exil des Tamouls du Sri Lanka ${ }^{10}$ qui a comme effet l'élaboration d'un espace politique en France dirigé contre le régime politique du Sri Lanka. Cet espace politique qui prend place dans des attitudes revendicatives et dans un projet, l'Eelam tamoul ${ }^{11}$, génère une politisation de la vie communautaire qui préserve les modèles culturels et les liens sociaux hérités de la communauté d'origine. Cet espace politique au travers une panoplie d'associations plus ou moins liées aux LTTE développe une façon particulière d'être tamoul en France. Il limite également les effets déstructurants de l'acculturation au prix d'une agrégation/ségrégation qui règle le jeu des proximités et des distances avec la société environnante.

Stéphane Dufoix nomme l'existence d'un tel espace « exopolitie » (2000, p. 47). Il s'agit d'un « espace social dans lequel le positionnement des différents groupes les uns par rapport aux autres leur donne un sens politique »(Dufoix, 2000, p. 159). Et c'est bien cette exopolitie qui distingue les Tamouls du Sri Lanka des autres groupes Tamouls en France.

\section{La mise en place d'un nationalisme à longue distance}

Il ne faut pas oublier que si les Tamouls du Sri Lanka ne sont présents en France que depuis la fin des années 1970, début des années 1980, c'est que le contexte de leur migration est diamétralement différent de celui des autres composantes de l'ensemble " Tamoul ». Ce sont majoritairement des réfugiés politiques. La nature de cette migration a des répercussions en termes de mobilisation, de revendication et d'idéologie. Elle contribue à former une communauté unie et relativement fermée. Cinq éléments liés au 
conflit au Sri Lanka génèrent des conditions sociales, spatiales et politiques particulières, qui ont progressivement conduits à la formation d'intenses liens communautaires.

- Premièrement, le flot de demandeurs d'asile à la fin des années 1970 dans les pays occidentaux a contribué à l'établissement de pôles diasporiques. Cette diffusion spatiale s'est faite à diverses échelles : pays, villes et aires urbaines.

- Deuxièmement, ces polarités ont été progressivement renforcées par les réseaux sociaux établis par les demandeurs d'asile.

- Troisièmement, ces arrivées étant de plus en plus importantes, on a assisté à la formation d'ethnoterritoires et d'aires urbaines ethniquement spécialisées à l'instar des phénomènes que nous avons pu décrire concernant la Chapelle et la Courneuve.

- Quatrièmement, alors que les premiers migrants, du fait de leur faible nombre, se sont facilement mélangés aux autres groupes, les nouveaux arrivants n'entretiennent pas le même rapport à l'altérité.

- Enfin, si l'Inde durant les évènements de 1983 a soutenu les militants Tamouls de Sri Lanka, depuis les années 1990, la situation a changé. L'Inde n'est plus une terre d'asile pour les militants ; c'est ce qui a aussi motivé le changement de direction de la migration des Tamouls vers l'Ouest.

Ensemble, ces différents facteurs ont participé à entretenir tout un faisceau de liens entre les membres de ce segment diasporique, et ce malgré la dispersion. Mais surtout, ce qui catalyse l'émergence d'une conscience diasporique et qui permet de distinguer ce groupe des autres, c'est l'engagement politique qui se manifeste par l'existence d'associations (association des étudiants tamouls, des femmes tamoules, des jeunes tamouls...), de journaux et de comités. Trois journaux tamouls sont publiés à Paris. Il s'agit d'Eelamurasu (les tambours de l'Eelam), d'Eelanadu et d'Erimalaï. A cela s'ajoute un journal en ligne créé en octobre 2007 par le comité de coordination tamoul France (ou CCTF) : Naam, nous Tamouls de France.

Les discours et les pratiques politiques sont devenus des mécanismes de renforcement des liens avec le référent-origine, mais aussi des liens entre les divers points de fixation de la diaspora. Les expériences pré-migratoires ont contribué à forger une identité politique en France. En cela, nous pouvons considérer l'expérience migratoire selon deux cadres: comme un moyen de préserver la culture, permettant aux Tamouls d'être Tamouls dans un environnement non-tamoul ; ou comme une façon d'être attaché au pays d'origine par la création d'un espace politique original dont la finalité serait le changement social et un hypothétique retour au Sri Lanka. Cependant, préciser que c'est parce que ce sont des réfugiés politiques que les Tamouls Sri Lankais sont engagés politiquement est une tautologie. Il est préférable de ne pas fixer les migrants tamouls dans les raisons réelles ou supposées de leur départ mais de les définir par les espaces sociaux successifs dans lesquels ils interviennent (Dufoix, 2000, p. 160). Ces espaces sociaux sont les enveloppes de mouvements revendicatifs dont le but est de permettre la mise en place de changements radicaux dans le pays d'origine.

Ainsi, cet espace politique dessine les contours d'un attachement diasporique et se caractérise par la présence de trois espaces sociaux successifs d'intervention des acteurs (un noyau dur, une arrière garde et l'ensemble des membres potentiels). 


\section{Les structures de « l'exopolotie »}

31 Comme a pu le constater Fuglerud à propos de la Norvège, le noyau dur de cette exopolitie est relativement peu important.

Figure 4 - Les structures de l'exopolitie

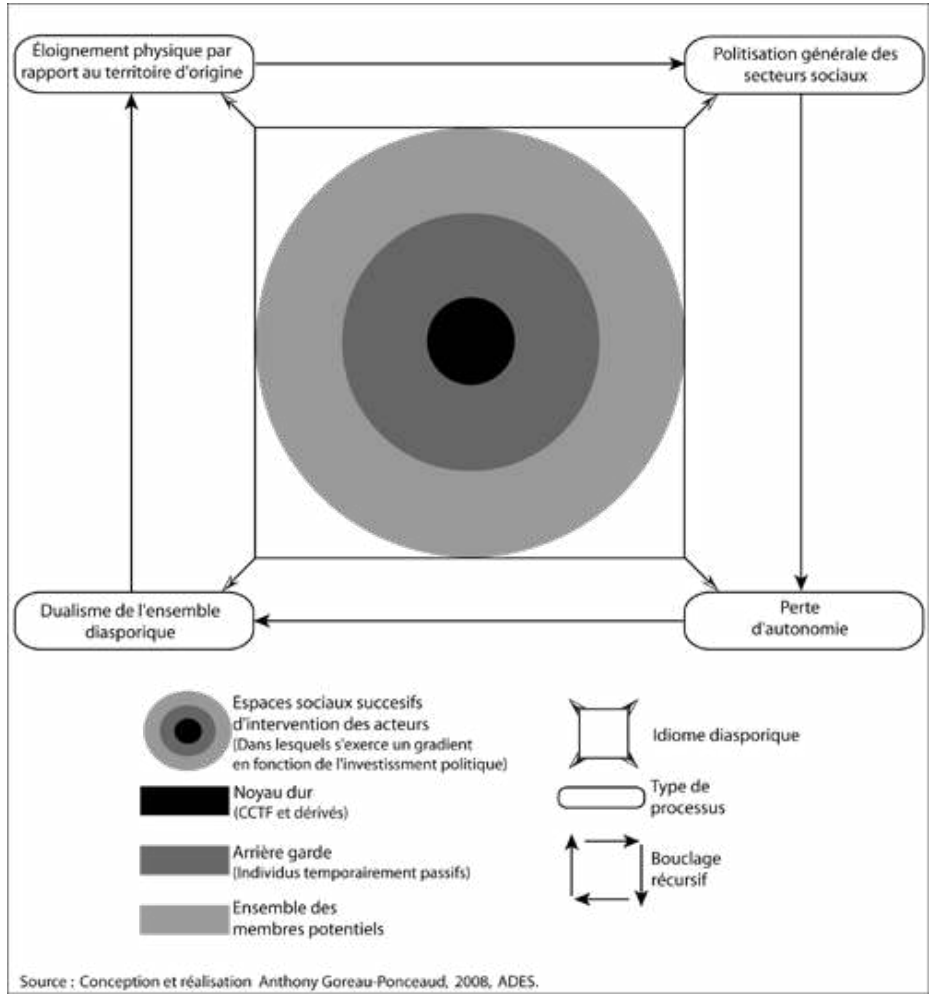

Il notait en 1999 que 5 à $10 \%$ des Tamouls Norvégiens sont actifs dans les organisations politiques de la diaspora, et que seulement une part restreinte de ce faible pourcentage supporte les actions des LTTE $(1999$, p. 85$)$. On peut faire un constat similaire en ce qui concerne les Tamouls de France. Sur l'ensemble des personnes que nous avons interrogées (221 en Île-de-France), seulement six se déclarent appartenir au CCTF. Bien que ce noyau dur soit caractérisé par sa faiblesse numérique, il n'est pas moins vrai qu'il influence l'ensemble des autres sphères sociales et se constitue en véritable groupe de pression en France, à tel point qu'il semble être l'unique représentant des Tamouls en France. La branche française du Comité de coordination des Tamouls (CCT) a été créée à Paris en 1981 sous un statut associatif. Ses membres ont, au cours des années 1990, réorienté leurs activités en substituant à l'aide administrative et sociale aux primoarrivants, une approche culturelle et communicationnelle auprès des familles tamoules anciennement installées (cours de langue tamoule et de civilisation, soutien scolaire, encadrement de la jeunesse par le biais des temples communautaires). Cette réorientation s'est concrétisée par la mise en place de tout un ensemble d'associations de contrôle communautaire. 


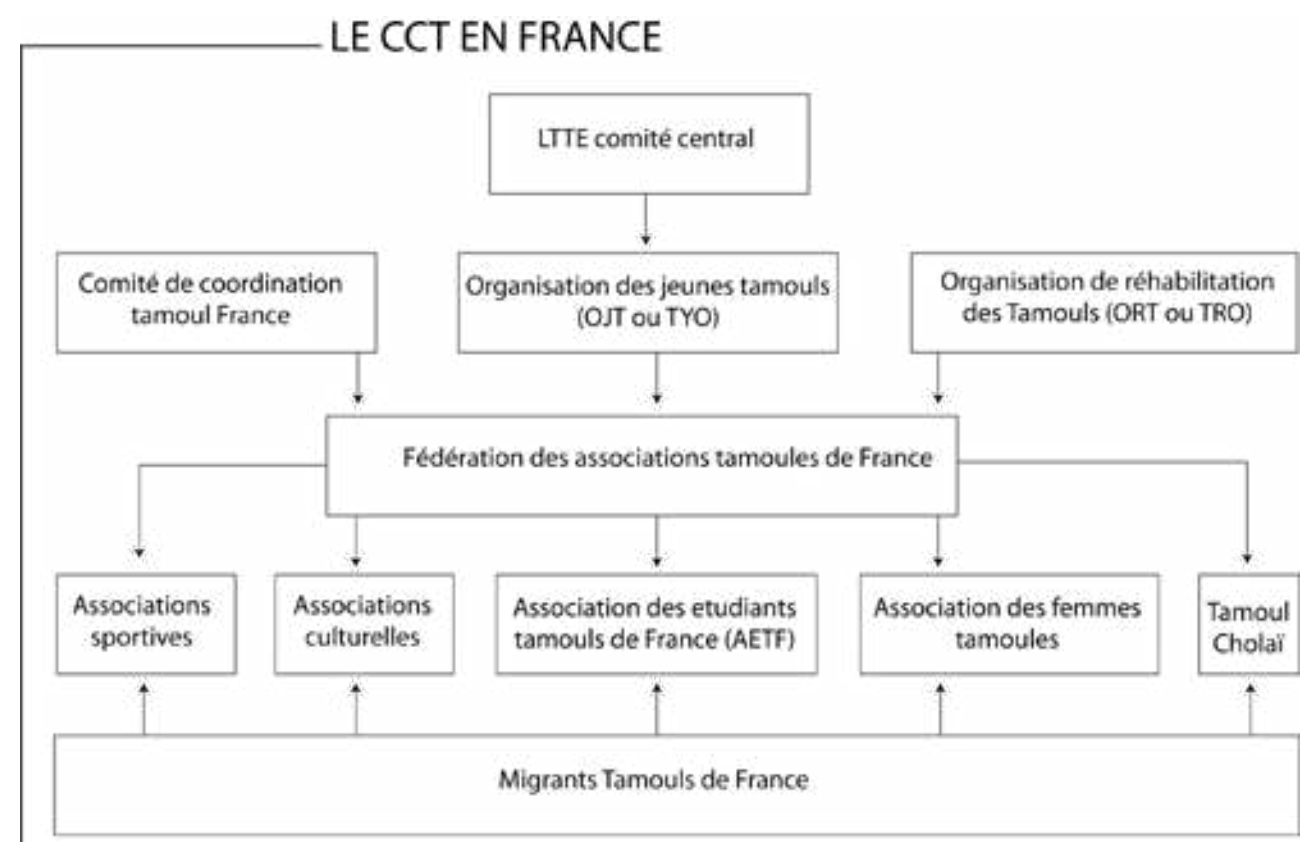

Conception et réalisation : Anthony Goreau-Ponceaud, 2008, ADES

Texte 2 - Le CCT

« En fait, il faut imaginer le CCT comme une maison mère. C'est un peu comme pour les
grandes entreprises internationales. Après il y a la FTF, c'est la fédération tamoule de
France, en gros elle regroupe soixante et une associations tamoules partout en France. À
cela s'ajoutent l'association des femmes tamoules, dont la responsable est Madame
Mougunthy, l'OJT, ou organisation des jeunes tamouls, dont le responsable est Monsieur
Mahinthan, la fédération sportive tamoule qui regroupe entre 30 et 40 fédérations
sportives, et des fédérations culturelles qui organisent des spectacles de danses et de
musique. Il y a aussi la jeunesse étudiante tamoule ainsi que l'association Tamoulcholai
qui regroupe plusieurs écoles de tamoul, de soutien scolaire en français, de musique et de
danse. Vous voyez, c'est une organisation très complète ».
Extrait d'un entretien mené en 2008, au siège du CCT au 341, rue des Pyrénées dans le 19e
arrondissement de Paris.
qui comme son nom le laisse à penser coordonne l'ensemble des actions. Cet emboittement permet sans doute de brouiller les pistes et de ne pas être étiqueté trop rapidement et trop ouvertement pro LTTE, tant par les Tamouls que par les institutions françaises. D'autant plus que les LTTE, ont été inscrits en 2006 sur la liste des organisations terroristes par l'Union Européenne.

Ainsi, le rôle assigné au secteur associatif est d'encadrer la jeunesse tamoule en s'assurant de sa socialisation à la culture tamoule et, dans le même temps, de son soutien à la cause. De plus, l'ensemble de ces associations sert de relais au CCTF dans ses actions de revendications politiques. Le CCTF voit dans ces associations un moyen de sensibiliser les jeunes générations à l'histoire du conflit, et pourquoi pas un moyen de recruter de nouveaux militants. Toutefois, les actions menées par ce noyau dur suscitent de multiples 
inquiétudes au sein de la société française. Ainsi, en avril 2007, des dizaines de militants présumés des LTTE ont été arrêtés au cours de plusieurs « descentes » effectuées par la police française en région parisienne. Les établissements visés par la police étaient le comité de coordination tamoul, des magasins et les logements de militants présumés du LTTE en France.

Cet épisode s'est conclu par une manifestation (entre 400 et 500 personnes se rassemblèrent) non autorisée qui eut lieu le 9 avril 2007 au Trocadéro afin de protester contre les mesures de répression prises contre les membres du CCT.

Ces évènements montrent que ce noyau dur centré sur le CCTF, est à la base de trois processus au sein de la communauté tamoule d'origine sri lankaise $:$ il a d'abord conduit à la politisation générale des secteurs sociaux liés à cette même communauté, pour générer dans un second temps une perte d'autonomie de cette dernière (toutes les actions entreprises pour et envers la communauté doivent avoir l'assentiment du CCTF), pour au final engendrer un dualisme au sein de l'ensemble de la diaspora. En effet, le CCT crée une ligne de clivage entre ceux qui veulent soutenir la cause et les autres, attachés à leur pays mais voulant rester en dehors de cette « omnipolitisation» (Sriskandarajah, 2005, p. 496). À terme, cet engagement politique a comme corollaire de créer un clivage entre ceux qui supportent les actions des LTTE et ceux qui les répriment et les condamnent.

Derrière ce noyau dur on trouve tout de même une arrière garde constituée par un public plus vaste. C'est cette population - difficile à évaluer numériquement - qui se rend aux manifestations diverses organisées par le CCTF. Nous la nommons arrière garde, car audelà de son apparente passivité, elle constitue pour le CCTF un groupe de population acquis à la cause et facilement mobilisable. Cette population est séduite par les discours, les évènements et les initiatives entreprises par et à travers ce nationalisme tamoul. Elle est capable de se mobiliser lors d'évènements particuliers, tels que Pongu Tamil (célébration pour l'autodétermination du peuple tamoul, la célébration du 18 juin 2008 a rassemblé plus de 7000 personnes à Paris) et Maaveerar Naal (journée des martyrs), qui permettent en retour de consolider ce corps social en lui fournissant des bases et arguments idéologiques (Ma Mung, 1995).

39 Ainsi, ces deux espaces sociaux successifs dessinent les contours d'une appartenance diasporique que l'on pourrait qualifier de nationale (Shukla, 2003, p. 252). En effet, le CCTF, arrive à mobiliser dans ces espaces politiques trois types d'éléments qui parviennent à définir ce qu'il entend par cette appartenance : un élément cognitif (connaître les limites de l'Eelam Tamoul), un élément affectif (en faisant appel aux souvenirs des morts lors des cérémonies collectives) et un élément instrumental. Il permet aux Tamouls d'être là-bas tout en étant de l'ici, d'être tamoul tout en étant hors du Tamil Nadu: le CCT contribue à l'émergence d'un imaginaire diasporique transnational fondé sur une dimension essentiellement politique.

Le dernier cercle de cette exopolitie est celui représenté par les membres potentiels, ceux qui peuvent être concernés par ces revendications. En dehors de la communauté tamoule du Sri Lanka, peu de personnes se sentent touchées par ces revendications politiques. Les membres sont à rechercher du côté des personnes encore rétives au mouvement, insensibles à la cause. Néanmoins, même si cet activisme politique crée un dualisme au sein de la diaspora tamoule, que l'on soit pour ou contre les préoccupations liées aux LTTE, les comités de coordination tamouls qui asphyxient l'espace diasporique, ont contribué à l'émergence d'une identité ethnique qui a pour but d'exalter une identité tamoule. Dans ce cas, les relations triadiques entre le pays d'origine, la diaspora et le pays 
d'accueil sont perturbées par les LTTE et les CCT qui prennent place dans cet ensemble. De plus, les pratiques politiques de la diaspora ne s'orientent pas vers un pays d'origine qui correspondrait à l'État-nation d'autrefois, mais vers une idéologie, celle de l'Eelam Tamoul.

Ainsi, l'exopolitie assure un type d'autonomie à la diaspora : celle de savoir maintenir sa propre spécificité par rapport à la société française. D’ailleurs, le succès de cette politisation tient en la capacité du noyau dur à importer l'idéologie de l'Eelam tamoul par les canaux de la diaspora en recrutant au sein de cette dernière des membres capables de véhiculer le message.

Mais, elle ne permet pas de choisir librement ses stratégies d'intégration et ses propres critères d'identification et de socialisation qui lui permettraient de prendre ses distances à l'encontre de la culture d'origine. Un des traits marquant de cet activisme politique est la prédominance de la langue tamoule et la volonté de maintenir une culture tamoule, et de montrer au monde la grandeur et la spécificité de cette culture. Et même si ces revendications politiques ne sont pas partagées par l'ensemble des membres de la communauté - tous les Tamouls de Paris ne sont pas passionnés et mus par des pensées sécessionnistes - elles concourent à renforcer une unité communautaire et à affirmer une ethnicité. Enfin, cette omnipolitisation du communautaire permet de comprendre la configuration des manifestations tamoules qui ont eu lieu au mois de janvier et avril de cette année où aucune banderole et slogan n'était affiché contre les exactions commises par les Tigres.

\section{De Gaza à Jaffna : vers une importation du conflit ?}

Du 27 décembre 2008 au 21 janvier 2009 s'est déroulée une offensive militaire à Gaza. Parallèlement, durant cette même période a eu lieu au Sri Lanka, et particulièrement dans la partie Nord, une offensive militaire intense et meurtrière menée par Colombo contre la rébellion des Tigres tamouls. Dans les deux cas, ces conflits ont engendré en France des réactions qui témoignent de l'intrication du local et du global. En effet, on a pu assister à la multiplication des manifestations de soutien dans l'espace public français : la communauté tamoule en particulier, a organisé au mois de janvier trois grandes manifestations ${ }^{12}$ et la dernière en date au mois d'avril a débouché sur l'interpellation de 210 personnes.

Le quartier de la Chapelle, durant ces manifestations, servait de tribune publique d'où partaient des messages à l'encontre, d'une part, des exactions et des massacres commis par l'armée gouvernementale envers les civils, et d'autre part, vis-à-vis de la faible médiatisation et préoccupation du conflit en France. Plus qu'un moyen d'alerter l'opinion publique, ces mobilisations étaient pour la jeune génération tamoule un moyen de prendre la parole et de se faire l'écho de la création d'un Etat indépendant au Sri Lanka pour veiller au respect des droits de cette minorité.

Simultanément, au cours de ce même mois de janvier, des démonstrations de soutien pour les Palestiniens ou pour les Israéliens avaient quotidiennement lieu en France. Le service de protection de la communauté juive, chargé sous l'égide du conseil représentatif des institutions juives de France, de recenser les actes antisémites en lien avec le Ministère de l'Intérieur, a alerté l'opinion publique d'une recrudescence des actes racistes et antisémites en France. C'est à partir de ce constat qu'un journaliste ${ }^{13} \mathrm{du}$ Monde a parlé d'une importation du conflit israélo-palestinien en France. Plus qu'une importation du 
conflit, on assistait donc à un accroissement des tensions communautaires entre arabes musulmans et juifs. Peut-on dresser le même constat dans le cas du conflit à Sri Lanka ? Y a-t-il depuis cette offensive militaire des tensions entre cinghalais et tamouls?

Il n'existe pas un tel service dans le cas des Tamouls. Par contre, ce que l'on peut constater, c'est que le seul commerce, ouvert en 2006 par un Cinghalais dans le quartier le Chapelle, a été détruit durant ce même mois de janvier.

\section{Conclusion - Pour une reconnaissance dans l'espace national ?}

Les Tamouls sri lankais s'efforcent d'assurer le lien avec leur référent-origine par la création de saillances identitaires qui remplissent des fonctions à la fois économiques, sociales, politiques, culturelles et cultuelles. Ces lieux particuliers, tels que la Chapelle, sont l'expression de la vitalité de cette communauté. Ce quartier est un territoire où se jouent, se construisent et s'inventent des logiques identitaires participant à la construction d'un chez-soi déterritorialisé. En même temps, une telle concentration urbaine est un lieu privilégié d'échange à l'intention des membres de la communauté bien sûr, mais aussi à l'intention de la société française et des autres minorités nationales ou non. Et, par le dynamisme de ses réseaux, les Tamouls sri lankais assurent pour les autres groupes tamouls, à travers ce patrimoine communautaire, la mise en place d'une identité renaissante, non pas à l'identique, en descendance, mais dans un cadre de placement qui est justement celui d'une diaspora dont les contours et le cœur même sont problématiques. Et, si la figure de l'autre est nécessaire pour s'affirmer soi-même et pour fixer des frontières spatio-culturelles qui permettent de se reconnaître (Barth, 1995) via un acte de marquage territorial, cette identité locale ne semble prendre sens que par rapport à une histoire mondialisée, celle d'un conflit sanglant, qui passe par la mise en place d'une exopolitie.

Cette dernière organise la visibilité des Tamouls en Île-de-France. Mais que visent réellement les cadres du CCTF lorsqu'ils exigent par ces manifestations d'être visibles dans l'interaction? S'agit-il d'une étape préliminaire à leur reconnaissance dans l'espace national?

$\mathrm{Au}$ final, cette visibilité tamoule pose de nouveaux défis et nous oblige à repenser la question des fondements légitimes de la citoyenneté et de la relation entre le citoyen et l'Etat, la Nation ou la nationalité.Cette exopoltie témoigne de la capacité des Tamouls du Sri Lanka à jouer sur plusieurs espaces. A tel point qu'ils paraissent être ni d'ici, ni de làbas, ni d'ailleurs, mais d'ici de là-bas et d'ailleurs à la fois. Ils évoluent dans une position de semi-intériorité, semi-extériorité. Parfaitement intégrés économiquement, socialement et même politiquement (aux dernières élections municipales, sept candidats tamouls originaires du Sri Lanka ont été élu en Île-de-France) les Tamouls du Sri Lanka apparaissent pourtant parfaitement «inassimilables $»^{14}$, évoluant dans une position d'impossibilité. Ils sont doublement absents, au lieu d'origine et au lieu d'arrivée. 


\section{BIBLIOGRAPHIE}

Anderson, B, 2002. L'imaginaire national : réflexions sur l'origine et l'essor du nationalisme. Paris, La Découverte, 207 p.

Bastenier A, 2004. Qu'est-ce qu'une société ethnique ? Ethnicité et racisme dans les sociétés européennes d'immigration. Paris, PUF, 346 p.

Bourdieu, P, 1999. Préface, in Sayad A, La double absence. Des illusions de l'émigré aux souffrances de l'immigré, Paris, Seuil.

Clifford, J, 1997. Routes: travel and translation in the late twentieth century. Cambridge, Harvard university press, $408 \mathrm{p}$.

Cohen, R, 1997. Global diasporas. An introduction. Londres, Routledge, 228 p.

Dequirez, G, 2007. Tamouls sri lankais : le Little Jaffna de La Chapelle. Hommes et migrations, $\mathrm{n}^{\circ}$ 1268-1269, p. 102-118.

Dufoix, S et Foucher, V, 2007. Les Petites Italies (et les autres...). Éléments de réflexion sur la notion d'ethnoterritoire, in Blanc-Chaléard M-C et al, Les Petites Italies dans le Monde, Rennes, PUR, p. 423-436.

Dufoix, S, 2000. Fausses évidences. Statut de réfugié et politisation. Revue européenne des migrations internationales, vol16, $\mathrm{n}^{\circ} 3, \mathrm{p} \cdot 147-164$.

Etiemble, A, 2003. Les Tamouls du Sri Lanka et l'exil en France : sens de la migration et dynamique de la diaspora. Les cahiers du CERIEM, vol. 11, p. 35-52.

Etiemble, A, 2002. Les Tamouls du Sri Lanka en France. Les arcanes de la « bonne éducation ». Ville, école, intégration, enjeux : nouvelles migrations, nouvelles formes de migrations, vol. 131, p. 176-190.

Fuglerud, 2001. Time and space in the Sri Lanka Tamil Diaspora. Nations and nationalism, vol. 7, $\mathrm{n}^{\circ} 2, \mathrm{p} .195-213$.

Fuglerud, 1999. Life on the outside. The Tamil Diaspora and long distance nationalism. Londres, plutopress, $203 \mathrm{p}$.

Glissant, E, 1996. Introduction à la poétique du divers. Paris, Gallimard, 144 p.

Goreau-Ponceaud, A, 2008. La diaspora tamoule : trajectoires spatio-temporelles et inscriptions territoriales en Île-de-France. Thèse de doctorat, université de Bordeaux, $426 \mathrm{p}$.

Goreau-Ponceaud, A, 2007. Bhangrâ et imaginaire de diaspora. Copyright volume ! vol. 6, $n^{\circ} 1 / 2$, p. 33-45.

Halbwachs, M, 1994. Les cadres sociaux de la mémoire. Paris, Albin Michel, 367 p.

Honneth, A, 2006. La société du mépris. Vers une nouvelle théorie critique. Paris, La Découverte, $349 \mathrm{p}$.

Lussault, M, 2007. L'homme spatial. La construction sociale de l'espace humain. Paris, Seul, 363 p. Ma Mung, E, 1995, Non lieu et utopie : la diaspora chinoise et le territoire, in Bruneau M, Diasporas. Montpellier, GIP Reclus, p. 163-174. 
McDowell, C, 1996. A Tamil asylum Diaspora. Sri Lankan migration settlement and politics in Switzerland. Royaume-Uni, Bergham Books, 308 p.

Meyer, E, 2001. Sri Lanka, entre particularismes et mondialisation. Paris, La Documentation Française, $184 \mathrm{p}$.

Raulin, A, 2000. L'ethnique est quotidien. Diasporas, marchés et cultures métropolitaines. Paris, L'Harmattan, $229 \mathrm{p}$.

Servan-Schreiber, C et Vuddamalay, V, 2007. Les étapes de la présence indienne en France. Hommes et migrations, $n^{\circ} 1268-1269$, p. 8-23.

Vidal, D, 1997. Empirisme et croyance dans l'hindouisme contemporain : quand les dieux boivent du lait. Annales, vol. 52, $n^{\circ}$ 4, p. 881-915.

\section{NOTES}

1. Nous reprenons ici la distinction établie par Bastenier (2004).

2. L'ensemble de cet article s'appuie sur des études ethnographiques menées sur plusieurs sites, à la fois en Île-de-France et en Inde, mêlant questionnaires et entretiens sur un échantillon composé dans sa totalité de 523 personnes (284 enquêtes en Île-de-France, 239 en Inde). Parmi les 284 enquêtés d'île-de-France, 221 d'entre eux étaient originaires du Sri Lanka, 22 de la Réunion et de Maurice, 12 des Antilles et 29 de Pondichéry. Parmi les 239 personnes interrogées en Inde du Sud (au Tamil Nadu, dans les districts de Ramanathapuram et de Naggapatinam, et dans le territoire de Pondichéry), 164 étaient des Français d'origine pondichérienne, 22 des Tamouls ayant de la famille installée en Île-de-France et 53 des réfugiés sri lankais fuyant par bateau le Sri Lanka.

3. Parmi notre échantillon de 523 personnes, un peu moins de $62 \%$ d'entre elles se disent hindoues, $37 \%$ chrétiennes et $1 \%$ musulmanes.

4. L'Eelam tamoul ou Tamil Eelam est le nom donné par les LTTE (Liberation Tigers of Tamil Eelam) au territoire revendiqué par ces derniers au Sri Lanka dans le cadre de la guerre civile.

5. La typologie de la demande sri lankaise est constante : il s'agit à $90 \%$ de Tamouls. Près de la moitié d'entre eux en 1994 avait entre 20 et 29 ans. En 2006, l'âge moyen de la première demande d'asile était de 31.8 ans (OFPRA). L'âge moyen des Tamouls sri lankais que nous avons interrogé est de 35.4 ans. Les évolutions ont concerné, en particulier, l'origine socioprofessionnelle et le sexe. Il semble que le niveau d'étude des candidats à la migration a eu tendance à baisser au fil du temps. De plus, la demande essentiellement masculine jusque dans les années 1980 s'est féminisée pour atteindre 30\% des premières demandes d'asile en 2006 et $37 \%$ du nombre des réfugiés statutaires sri lankais en France (rapports de l'OFPRA).

6. On peut aussi noter, à partir de nos entretiens, le rôle important joué par la CIMADE (Comité Intermouvements Auprès Des Evacués) dans l'accueil de ces primo-arrivants, principalement dans son rôle d'intégration via l'apprentissage du français. Nombre de personnes interrogées ont pris des cours de français par la CIMADE avant de faire leur première demande de naturalisation. 7. On recense selon les estimations officielles 105 camps de réfugiés dans le Tamil Nadu dont les plus importants sont situés dans les districts du Sud de l'État. Ces camps accueillent des populations civiles du Sri Lanka qui fuient la guerre et l'oppression. D'après les informations que nous avons obtenues en 2007 lors de nos enquêtes dans la région, ces camps accueilleraient environ 60000 individus, soit plus de 17000 familles. Nous avons trouvé un écho à ces informations données dans l'édition de The Hindu du 19 février 2007. Les familles qui arrivent dans ces camps sont principalement originaires des villes de Jaffna, Trincomalee, Mannar et Vavuniya. La traversée entre les différents sites de débarquement en Inde et Talaimannar coûte 
entre 5000 et 10000 roupies indiennes. De Mannar à Danushkodi, le prix de la traversée est fixée a approximativement 5000 roupies par personne.

8. Cette population tamoule d'origine vietnamienne est en réalité une caste de marchands qui a longtemps servi de relais dans la création et l'ouverture des établissements commerciaux sudasiatiques (mauriciens, sri lankais, pakistanais, indiens et bangladais) en France.

9. Déjà, dans l'édition du Monde du 24 octobre 1992, Jean Louis Perrier utilisait ce terme pour décrire cette concentration commerciale. Après il a été largement popularisé jusqu'à être substitué par l'étiquette « little Jaffna » au cours des années 2000 (Goreau-Ponceaud, 2008).

10. Depuis plus de trente ans, le Sri Lanka connaît une guerre entre le mouvement séparatiste tamoule et les forces majoritairement cinghalaises au pouvoir à Colombo. Ce séparatisme, expression de la mobilisation d'une minorité autochtone autour de revendications politiques et linguistiques est le fruit d'une entreprise politico-militaire fondée sur une puissante organisation : les LTTE (Liberation Tigers of Tamil Eelam) ou Tigres tamouls (Meyer, 2001).

11. L'Eelam tamoul ou Tamil Eelam est le nom donné par les LTTE (Liberation Tigers of Tamil Eelam ) au territoire revendiqué par ces derniers au Sri Lanka dans le cadre de la guerre civile.

12. Il y a eu des manifestations le 4 janvier rassemblant plus de 10000 personnes, le 7 et le 14 . Ces manifestations ont été organisées par le CCT et l'OJTF (Organisation des Jeunes Tamouls de France). Elles se sont déroulées devant l'Assemblée Nationale et à la Place de la République. Des manifestations ont eu lieu le même jour dans toute l'Europe. A chaque fois, elles étaient organisées à l'initiative du CCT. Lors de ces évènements, on pouvait lire sur certaines pancartes Sri Lanka = Gaza.

13. Sabot Antonin., Y a-t-il une importation du conflit israélo-palestinien en France? Le Monde, 15 janvier 2009.

14. «L'immigré est atopos, sans lieu, déplacé, inclassable [...] ni vraiment du côté du Même, ni totalement du côté de l'Autre, il se situe en ce lieu «bâtard » dont parle aussi Platon, la frontière de l'être et du non être social » (Bourdieu, 1999:12).

\section{RÉSUMÉS}

Les manifestations organisées par la communauté tamoule sri lankaise en janvier et en avril 2009, qui se sont déroulées dans le quartier la Chapelle à Paris, peuvent servir de point de départ pour analyser ce que l'on entend par diaspora tamoule. Ces évènements mettent en lumière une visibilité qui vise une reconnaissance dans l'espace public français. Reconnaissance qui passe par un besoin d'identification dans l'interaction. Cet article vise à expliciter les éléments constitutifs de cette visibilité tout en s'interrogeant sur une des spécificités de cette population: sa politisation.

The demonstrations organized by the Sri Lankan Tamil community in January and April 2009 held in the la Chapelle area in Paris, can be a starting point to analyze what is meant by a Tamil Diaspora. These events highlight a visibility that aims at recognition in the French public space. This recognition goes with a need for identification in the interaction. This article aims to explain the components of this visibility while questioning one of the specificities of this population: its politicization. 


\section{INDEX}

Mots-clés : Diaspora tamoule, nationalisme, identité, territoire, réseau

Keywords : Tamil Diaspora, nationalism, identity, territory, network

\section{AUTEUR}

\section{ANTHONY GOREAU-PONCEAUD}

Anthony Goreau-Ponceaud (anthonygoreau@yahoo.fr) nest docteur en géographie. Il a soutenu sa thèse en 2008, à l'université de Bordeaux 3, sous la direction de Singaravelou. L'intitulé en est «La diaspora tamoule : trajectoires spatio-temporelles et inscriptions territoriales en île-deFrance ». Il est membre de l'UMR ADES et travaille actuellement, en collaboration avec l'équipe diaspora de l'Océan indien du CEIAS, sur les réseaux diasporiques indiens dans l'aire indanocéanique entre Afrique de l'Est, Sri Lanka et Inde. L'auteur a publié, entre autre, « la diaspora tamoule : lieux et territoires en Île-de-France » (2008), L'espace politique, $\mathrm{n}^{\circ} 4$, p.21-35; « De l'image au voyage : l'Inde sur la route de soi » (2008), Articulo, ${ }^{\circ} 4$. 